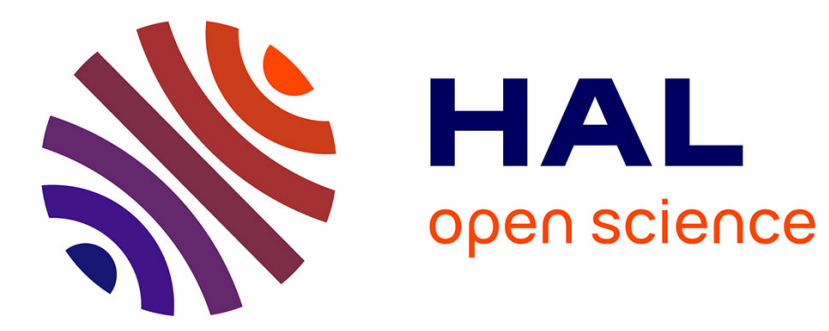

\title{
Evolution de la taille des grains du silicium polycristallin pendant des traitements thermiques ou oxydation
}

\author{
M. Lemiti, S. Audisio, C. Mai, B. Balland
}

\section{To cite this version:}

M. Lemiti, S. Audisio, C. Mai, B. Balland. Evolution de la taille des grains du silicium polycristallin pendant des traitements thermiques ou oxydation. Revue de Physique Appliquée, 1989, 24 (2), pp.133141. 10.1051/rphysap:01989002402013300 . jpa-00246035

\section{HAL Id: jpa-00246035 https://hal.science/jpa-00246035}

Submitted on 1 Jan 1989

HAL is a multi-disciplinary open access archive for the deposit and dissemination of scientific research documents, whether they are published or not. The documents may come from teaching and research institutions in France or abroad, or from public or private research centers.
L'archive ouverte pluridisciplinaire HAL, est destinée au dépôt et à la diffusion de documents scientifiques de niveau recherche, publiés ou non, émanant des établissements d'enseignement et de recherche français ou étrangers, des laboratoires publics ou privés. 
Classification

Physics Abstracts

$61.10 \mathrm{~F}-73.60 \mathrm{Hy}-81.60 \mathrm{j}$

\title{
Evolution de la taille des grains du silicium polycristallin pendant des traitements thermiques ou oxydation
}

\author{
M. Lemiti ( $\left.{ }^{1}\right)$, S. Audisio ( $\left.{ }^{2}\right)$, C. Mai $\left({ }^{3}\right)$ et B. Balland ( $\left.{ }^{1}\right)$ \\ $\left({ }^{1}\right)$ Laboratoire de Physique de la Matière (UA-CNRS 358), Bât. 502, $\left({ }^{2}\right)$ Laboratoire de Chimie industrielle, \\ Bât. 401, $\left({ }^{3}\right.$ ) Laboratoire GEMPPM (UA 341), Bât. 502, INSA-Lyon 20 av. Albert Einstein, 69621 Villeur- \\ banne Cedex, France
}

(Reçu le 11 décembre 1987, révisé le 21 juin 1988, accepté le 23 septembre 1988)

\begin{abstract}
Résumé. - Nous avons comparé l'évolution de la morphologie cristallographique de couches épitaxiques de silicium polycristallin (résistivité de $9 \times 10^{-3} \Omega . \mathrm{cm}$, épaisseur de $450 \mathrm{~nm}$, dépôt L.P.C.V.D. à $670^{\circ} \mathrm{C}$ par décomposition du silane) soumises pendant 6 à $180 \mathrm{~min}$, à un traitement thermique à température élevée (entre $900^{\circ} \mathrm{C}$ et $1050^{\circ} \mathrm{C}$ ) sous atmosphère soit inerte (argon), soit oxydante (oxygène sec). L'évolution de la taille des cristallites de la couche polycristalline a été mise en évidence par diffraction des rayons X. Dans les deux cas, on observe un accroissement (plus rapide lors d'une oxydation) de la taille des grains (de moins de $20 \mathrm{~nm}$ à plus de $70 \mathrm{~nm}$ ), correspondant à une cristallisation des zones amorphes, favorisée par la diffusion du dopant (phosphore) aux joints de grains et par l'existence de contraintes mécaniques. La vitesse de formation de l'oxyde est contrôlée par l'orientation cristallographique aléatoire des grains et la présence des joints de grains (diffusion intergranulaire et ségrégation des dopants). Le claquage destructif de l'oxyde n'est pas direct, on observe souvent une phase de préclaquage avec une auto-guérison. La rigidité diélectrique dépend du dopage et varie de $2 \mathrm{MV} / \mathrm{cm}$ à $5 \mathrm{MV} / \mathrm{cm}$ quand la température d'oxydation passe de $900{ }^{\circ} \mathrm{C}$ à $1000^{\circ} \mathrm{C}$.
\end{abstract}

\begin{abstract}
We compare the crystallographic morphology of epitaxial polycrystalline silicon layers $\left(9 \times 10^{-3} \Omega\right.$.cm resistivity, $450 \mathrm{~nm}$ thickness, L.P.C.V.D. at $670^{\circ} \mathrm{C}$ by silane decomposition) which undergo for 6 to $180 \mathrm{~min}$ a high temperature treatment $\left(900^{\circ} \mathrm{C}\right.$ to $1050^{\circ} \mathrm{C}$ ) in inert gas ambiance (argon) or oxidizing ambiance (dry oxygen). The crystallite size evolution of the polycrystalline layer has been studied by means of $\mathrm{X}$-Ray diffraction. In both cases, we find a grain size increase (faster in oxygen ambiance) (from 20 to more than $100 \mathrm{~nm}$ ), corresponding to the crystallization of amorphous zones enhanced by dopant diffusion (phosphorus) at grain boundaries and by mechanical stresses. As compared to single crystal, the oxide formation kinetics is controlled by the random crystallographic orientation of grains and by grain boundaries (intergrain diffusion and dopant segregation). The destructive breakdown of oxide is not direct. We often observe a pre-breakdown followed by self-curing. Dielectric rigidity depends on doping rate and varies from 2 to $5 \mathrm{MV} / \mathrm{cm}$ when the oxidation temperature steps from $900^{\circ} \mathrm{C}$ to $1000{ }^{\circ} \mathrm{C}$.
\end{abstract}

\section{Introduction.}

Dans la technologie microélectronique, le silicium polycristallin fortement dopé est actuellement le matériau de grille le plus utilisé. Il permet non seulement de réaliser les électrodes de grille, mais aussi les interconnexions dans les circuits M.O.S. intégrés et les dispositifs mémoires. L'oxydation du silicium polycristallin constitue une phase technologique importante, dans la fabrication des composants V.L.S.I. [1]. En effet, le film d'oxyde $\left(\mathrm{SiO}_{2}\right)$ obtenu sur des couches de silicium déposées est nécessaire, par exemple, pour assurer l'isolation inter-ligne, comme source de diffusion pour former des jonctions dans les dispositifs M.O.S. et dans les circuits intégrés bipolaires. Pour ces applications, deux ou trois couches de silicium polycristallin, séparées par des films d'oxyde sont de plus en plus fréquemment utilisées (dispositifs mémoires R.A.M. et P.R.O.M., dispositifs à couplage de charge). L'oxyde joue donc un rôle très important en tant qu'isolant dans les «structures multi-poly " (multicouches hétérogènes utilisées dans la réalisation de dispositifs mémoires).

Le développement de ces technologies repose donc en partie, sur l'obtention d'une couche d'oxyde 
isolante présentant une bonne rigidité diélectrique, qui serait comparable à celle de l'oxyde thermique obtenu sur du silicium monocristallin (champ de claquage de $5 \mathrm{MV} / \mathrm{cm}$ à $11 \mathrm{MV} / \mathrm{cm}$ ).

Actuellement, l'oxydation thermique de films minces de silicium polycristallin fortement dopé est très mal connue (cinétique d'oxydation, morphologie de l'oxyde et de l'interface, etc.). Notons que, sauf à très haute température, le modèle de Deal-Grove $[2,3]$ ne peut pas être utilisé pour décrire la croissance de l'oxyde [4]. La présence des joints et l'orientation aléatoire des grains jouent un rôle très important sur la cinétique d'oxydation [5], et par conséquent sur les propriétés diélectriques des couches.

Dans cette présente étude, nous avons suivi, en particulier, l'évolution de la taille des grains, de couches épitaxiques de silicium polycristallin soumises à un traitement thermique à température élevée (entre $900^{\circ} \mathrm{C}$ et $1050^{\circ} \mathrm{C}$ ) sous atmosphère soit inerte (argon), soit oxydante (oxygène sec) pendant des durées variables. La variation de la taille des cristallites de la couche du polycristal a été mise en évidence par diffraction des rayons $\mathrm{X}$. La cinétique d'oxydation du silicium polycristallin a été étudiée et comparée à celle du monocristal $[6,16]$. Des mesures électriques ont permis d'évaluer les qualités des couches d'oxyde et de relier celles-ci aux caractéristiques morphologiques du silicium polycristallin et de son oxyde (taille des grains, "rugosité » de l'interface et « pointes de champ » initiatrices du claquage diélectrique primaire).

\section{Procédure expérimentale.}

2.1 PRÉparation Des ÉChantillons. - Les échantillons ont été réalisés à partir de plaquettes de silicium monocristallin d'orientation (100), de type $\mathrm{P}$, dopé au bore avec une concentration de l'ordre de $10^{15} \mathrm{at} / \mathrm{cm}^{3}$. Après nettoyage et élimination de l'oxyde natif, une couche de silicium polycristallin a été déposée par décomposition thermique du silane (mélange gazeux de $\mathrm{SiH}_{4}(25 \%)$ et $\mathrm{N}_{2}(75 \%)$ ), sous une pression réduite de $100 \mathrm{~Pa}$ (L.P.C.V.D. Low Pressure Vapor Chemical Deposition $[7,25])$ et à une température de l'ordre de $670^{\circ} \mathrm{C}$. L'épaisseur de la couche déposée pour tous les échantillons étudiés est de $450 \mathrm{~nm}$. Le dopage des couches de silicium polycristallin se fait pendant le dépôt, par introduction de phosphine $\left(\mathrm{PH}_{3}\right)$ dans la phase gazeuse (dopage in situ). La concentration en phosphore est évaluée, par des mesures de résistivité, à $10^{19} \mathrm{at} / \mathrm{cm}^{3}$.

Ces films de silicium polycristallin ont été soumis à des traitements thermiques, sous pression atmosphérique, pendant différentes durées allant de $6 \mathrm{~min}$ à $3 \mathrm{~h}$, et à des températures de $900^{\circ} \mathrm{C}, 950^{\circ} \mathrm{C}$, $1000^{\circ} \mathrm{C}$ et $1050^{\circ} \mathrm{C}$. Certains ont été traités sous atmosphère inerte d'argon, et les autres sous oxygène sec.

2.2 MÉThodes anAlytiQues. - Les caractéristiques géométriques et électriques des couches de silicium polycristallin et des oxydes formés ont été mesurées par différentes techniques. L'ellipsométrie (mesure de l'indice de réfraction et de l'épaisseur des couches), la microscopie électronique à transmission $[9,18]$ (aspect des cristallites et de l'interface $\mathrm{SiO}_{2} /$ silicium polycristallin), la diffraction des $\mathrm{RX}$ (mesure de la taille des cristallites), la méthode des quatre pointes (résistivité des couches polycristallines) et la méthode du $I(V)$ (mesure de la rigidité diélectrique et du champ de claquage).

Ellipsométrie. - L'indice de la couche polycristalline a été déterminé pour des longueurs d'ondes se situant entre 240 et $700 \mathrm{~nm}$. Pour celle retenue, $\lambda=632,8 \mathrm{~nm}$, les parties réelle $n$ et imaginaire $k$, sont comprises respectivement entre 3,41 et 3,46 et, entre 0,643 et 0,885 . Les épaisseurs de silice sont mesurées pour cette même longueur d'onde en considérant un modèle à trois couches : air/oxyde/silicium polycristallin.

Microscopie. - Les analyses par microscopie électronique à transmission (M.E.T.) ont également permis d'évaluer l'épaisseur de la couche d'oxyde formée et d'observer la rugosité de l'interface et la structure des grains.

Pour les études par M.E.T. des coupes en sections droites ou parallèles à la surface ont été préparées. Les premières ont été obtenues en clivant des lamelles de $1 \mathrm{~mm}$, à partir des tranches, après métallisation par évaporation d'aluminium sur la surface de l'oxyde. Ces lamelles ont, ensuite, été collées face à face avec une résine «époxy », puis polies mécaniquement pour réduire l'épaisseur à $50 \mu \mathrm{m}$. Ensuite, elles sont exposées à un faisceau d'ions de basse énergie, afin d'obtenir un cratère puis un trou.

Diffraction RX. - Tous les échantillons traités ont été analysés par diffraction $\mathrm{X}$. Cette technique a permis de définir la taille des cristallites du silicium polycristallin avant et après traitement de recuit ou d'oxydation. Pour des tailles de grains inférieures à $100 \mathrm{~nm}$, on observe un élargissement très important des raies de diffraction. La largeur à mi-hauteur $B$, du pic de diffraction (voir Fig. 1a), est reliée à la dimension moyenne $D$ des grains par la relation de Scherrer $[8,10]$ :

$$
B=\frac{0,89 \lambda}{D \cos \theta}-b
$$

où $\lambda$ est la longueur d'onde du faisceau $\mathrm{X}, \theta$ la position angulaire de la raie, et $b$ la largeur « instrumentale », c'est-à-dire la largeur à mi-hauteur du pic de diffraction relatif à des grains de tailles supérieu- 


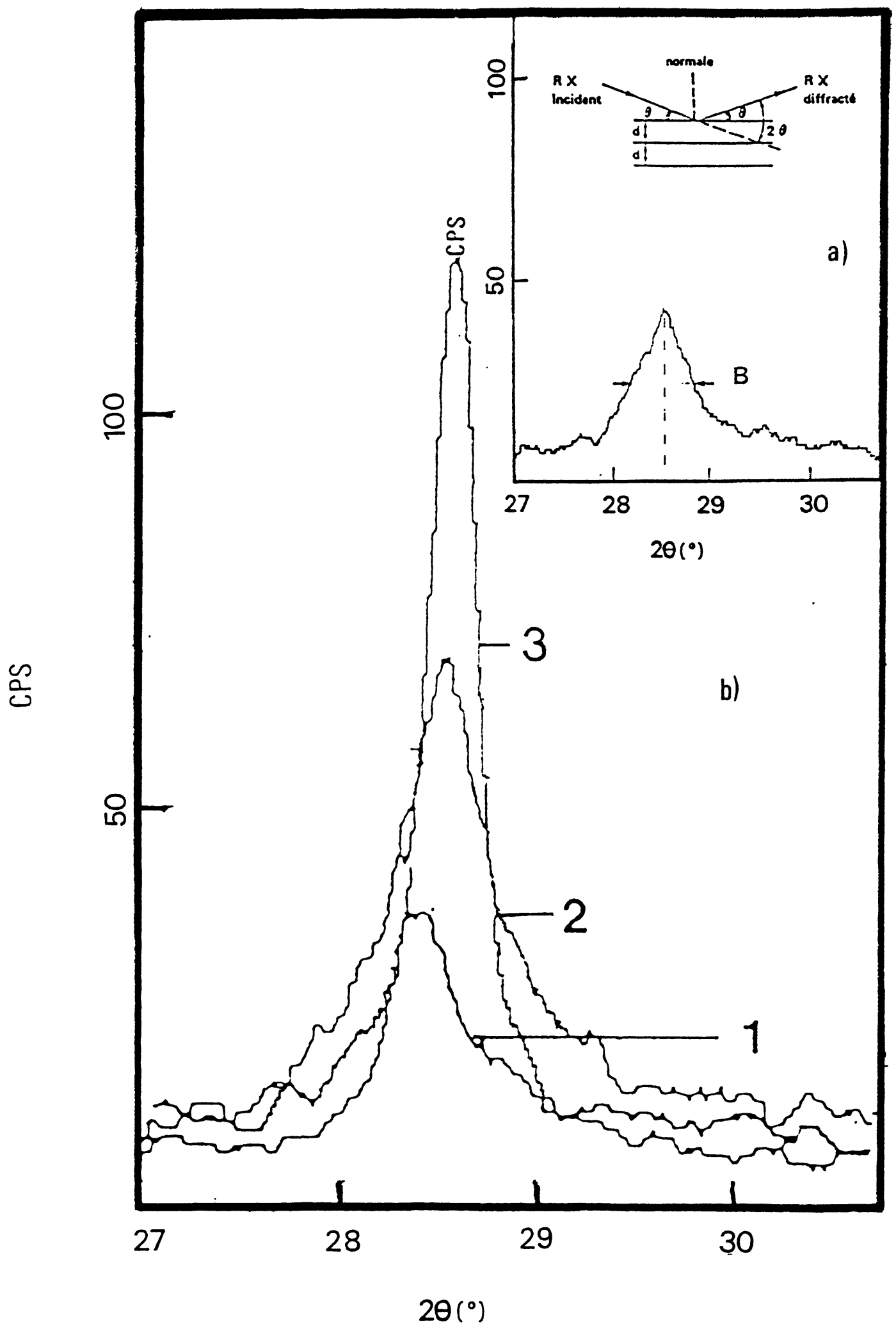

Fig. 1. - a) Méthode de détermination de la taille moyenne des grains par la diffraction des rayons X. La mesure de la largeur à mi-hauteur du pic de diffraction, par rapport au fond continu, permet de déterminer les tailles des cristallites ; b) Evolution du pic de diffraction du plan (111) du silicium polycristallin en fonction de la température : (1) non traité, (2) oxydation à $900^{\circ} \mathrm{C}$, (3) oxydation à $1050^{\circ} \mathrm{C}$.

(a) Method of the grain mean size determination by X-Ray diffraction. The measurement of diffraction peak mid height above steady bottom signal allows to determine crystallite grain sizes ; b) Diffraction peak evolution for (111) direction of polycrystalline silicon versus temperature : (1) no treatment, (2) $900{ }^{\circ} \mathrm{C}$ oxidation, (3) $1050{ }^{\circ} \mathrm{C}$ oxidation.] 
res à $100 \mathrm{~nm}$. Celle-ci a été mesurée à partir d'une poudre de silicium présentant des grains de l'ordre de $1000 \mathrm{~nm}$. On peut donc suivre, à partir de la mesure de $B$, l'évolution de la grosseur des grains pour $D$ compris entre $10 \mathrm{~nm}$ et $100 \mathrm{~nm}$. Par exemple, la figure $1 b$ représente la variation du profil de la raie (111) après différents traitements.

Nous avons analysé la raie correspondant à la famille de plans (111), celle-ci est la plus intense (hormis la raie (100)) et ce, quel que soit le traitement thermique appliqué. La raie (100) ne peut pas être utilisée, car la contribution du substrat monocristallin d'orientation (100) est largement dominante et masque celle de la couche polycristalline.

La résistivité des couches de silicium polycristallin. - Elle a été mesurée par la méthode des quatre pointes, pour évaluer la concentration en dopant (phosphore) à la surface, avant et après les différents traitements thermiques.

Les propriétés électriques des couches d'oxyde. Elles ont été analysées de manière à préciser les conditions opératoires optimales d'oxydation. Les mesures de conduction électrique et de champ de claquage sont faites sur des structures M.I.M. soit $\mathrm{Al} /$ oxyde/silicium polycristallin. Ces structures ont été préparées par dépôt, sur la couche de $\mathrm{SiO}_{2}$, de plots d'aluminium (évaporation sous vide), dont l'aire est de $7,85 \times 10^{-3} \mathrm{~cm}^{2}$, et l'épaisseur de $200 \mathrm{~nm}$. Une partie de la couche d'oxyde est ensuite éliminée par une attaque à l'acide fluorhydrique (HF) dilué ; un contact électrique est établi directement sur le silicium. La grille d'aluminium est soumise à une polarisation négative et l'intensité du courant de conduction en régime permanent (après extinction du régime transitoire [11]) est mesurée avec un picoampèremètre connecté entre le contact sur silicium polycristallin et la masse (point de potentiel le plus bas). L'oxyde est considéré comme définitivement détruit, lorsque l'intensité dépasse $100 \mu \mathrm{A}$.

\section{Résultats-discussion.}

3.1 CinÉtioue D’OXYdation. - La cinétique d'oxydation du silicium polycristallin et celle du silicium monocristallin sont décrites sur la figure 2 , qui représente la variation d'épaisseur de l'oxyde formé en fonction du temps et pour diverses températures $\left(900{ }^{\circ} \mathrm{C}, 950{ }^{\circ} \mathrm{C}\right.$ et $\left.1000^{\circ} \mathrm{C}\right)$. On note que la croissance de la couche de $\mathrm{SiO}_{2}$ est plus rapide sur le polycristal que sur le monocristal.

Si l'on se réfère aux travaux de différents auteurs [15-18] sur l'oxydation du silicium monocristallin, pour le domaine de température étudié compris entre $850^{\circ} \mathrm{C}$ et $1050^{\circ} \mathrm{C}$, les lois simples déduites du modèle de Deal et Grove, de type exponentiel,

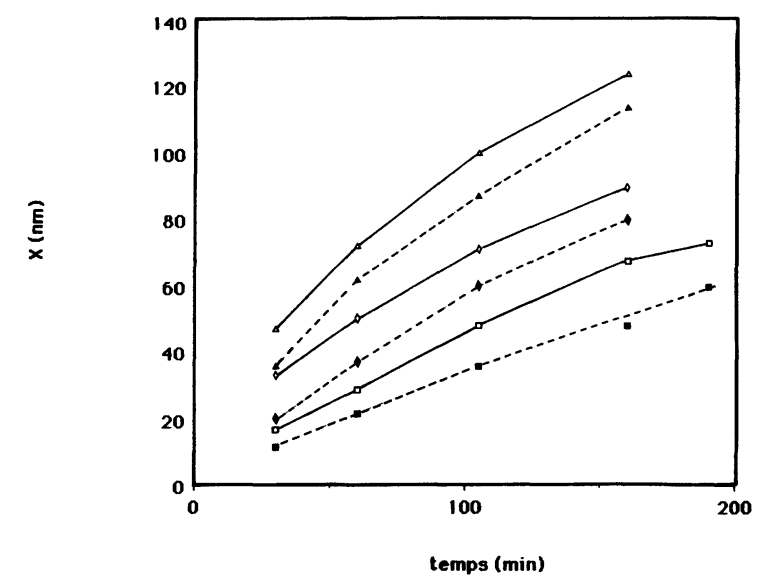

Fig. 2. - Cinétique d'oxydation du silicium polycristallin (-) et du silicium monocristallin (100) (---) : Variation de l'épaisseur de la couche d'oxyde thermique (sous $\mathrm{O}_{2} \mathrm{sec}$ et à $1 \mathrm{~atm}$.) en fonction de la durée du traitement, pour trois températures : $\quad T=900^{\circ} \mathrm{C}(\square), \quad T=950^{\circ} \mathrm{C}(\diamond)$, $T=1000^{\circ} \mathrm{C}(\triangle)$.

[Polycrystalline (-) and monocrystalline (---) (100) silicon oxidation kinetics : thermal (in dry $\mathrm{O}_{2}$ and atmospheric pressure) oxide layer thickness variation versus treatment duration, for three temperatures: $T=900^{\circ} \mathrm{C}(\square)$, $T=950^{\circ} \mathrm{C}(\diamond), T=1000^{\circ} \mathrm{C}(\triangle)$.]

linéaire ou logarithmique ne conviennent pas pour décrire la croissance de l'oxyde sur le silicium polycristallin $[12,14]$.

Cependant, pour expliquer les résultats que nous avons obtenus, une relation empirique de la forme $\mathrm{X}=\beta \cdot t^{\alpha}$, où $\beta$ exprime une vitesse d'oxydation (en nm. $\min ^{-\alpha}$ ) et $\alpha$ un coefficient dont la valeur est comprise entre 0,5 et 1 (cf. Tab. I), semble bien adaptée pour décrire l'évolution temporelle de l'épaisseur $\mathrm{X}$, de la couche de $\mathrm{SiO}_{2}$. On note que le paramètre $\alpha$ diminue et tend vers 0,5 quand la température augmente ; c'est-à-dire que la relation empirique $X=\beta \cdot t^{\alpha}$ se rapproche d'une loi de la forme $X=k t^{1 / 2}$ déduite du modèle de Deal et Grove et qui décrit l'oxydation du silicium monocristallin.

Tableau I. - Variation des paramètres empiriques $\alpha$ et $\beta$ en fonction de la température d'oxydation.

[Empirical parameters ( $\alpha$ and $\beta$ ) variation versus oxidation temperature.]

\begin{tabular}{rcc}
\hline$T\left({ }^{\circ} \mathrm{C}\right)$ & $\operatorname{Ln} \beta$ & $\alpha$ \\
\hline 900 & 2,19 & 0,84 \\
\hline 950 & 3,72 & 0,6 \\
\hline 1000 & 4,38 & 0,54 \\
\hline
\end{tabular}


La difficulté de modélisation de la vitesse de formation de l'oxyde sur le silicium polycristallin est due au grand nombre de facteurs intervenant dans le mécanisme de formation de $\mathrm{SiO}_{2}$ : l'orientation cristallographique des grains, la température d'oxydation, le dopage, etc. L'influence de ces paramètres est selon nous la suivante :

L'orientation cristallographique des grains du polycristal a une influence déterminante. Comme pour le silicium monocristallin, les plans (110) s'oxydent plus rapidement que les (100), et plus lentement que les (111). Cette orientation étant aléatoire, certains grains subissent une oxydation plus lente et émergent à l'interface silicium polycristallin/oxyde. Ils créent alors des protubérences, comme le montre l'analyse au M.E.T. (photo 1 de la Fig. 3) où, on observe une « ondulation » à l'interface $\mathrm{Si} / \mathrm{SiO}_{2}$, pour un échantillon oxydé à $950^{\circ} \mathrm{C}$ pendant $1 \mathrm{~h}$.

A basse température, l'oxydation est contrôlée par une réaction de surface régie par le mécanisme de diffusion préférentielle aux joints de grains. L'oxydation se produit plus rapidement au niveau de ces derniers (oxydation intergranulaire). De plus, comme dans le cas du silicium monocristallin, la présence d'une plus forte concentration de phosphore favorise la croissance de l'oxyde.

Par contre, à plus haute température, la diffusion volumique de l'espèce oxydante devient le processus prédominant, l'influence de l'orientation des grains et la présence des joints de grains est beaucoup moins importante. La vitesse d'oxydation du silicium polycristallin tend alors vers celle du monocristal.

3.2 TAILlE DES GRAINS. - Afin de faire la distinction entre l'effet du traitement thermique à température élevée, et celui de l'oxydation, nous avons suivi, par analyse en diffraction RX, l'évolution de la grosseur des grains du silicium polycristallin pendant un recuit sous argon, ou une oxydation sous oxygène sec.

Nous avons par ailleurs, évalué la taille moyenne des grains par M.E.T. (photo 2 de la Fig. 3). Par exemple, pour un échantillon oxydé à $1050^{\circ} \mathrm{C}$ pendant $30 \mathrm{~min}$, elle est de l'ordre de $91 \mathrm{~nm}$, avec une précision de $4 \%$. Cette valeur est en très bon accord avec celle obtenue par diffraction $X$ (soit $90 \mathrm{~nm}$ ), ce qui valide la technique $R X$ que nous avons utilisée.

La figure $1 \mathrm{~b}$ représente l'évolution de la raie de diffraction des plans (111), pour un échantillon non traité et pour deux échantillons oxydés l'un à $900^{\circ} \mathrm{C}$ l'autre à $1050^{\circ} \mathrm{C}$, pendant $1 \mathrm{~h}$. On note, un rétrécissement de la raie accompagné d'un accroissement de son amplitude et un déplacement vers les grands angles de diffraction.

Comme nous l'avons souligné, la largeur de la raie permet d'accéder à la taille des grains. Les échantil-

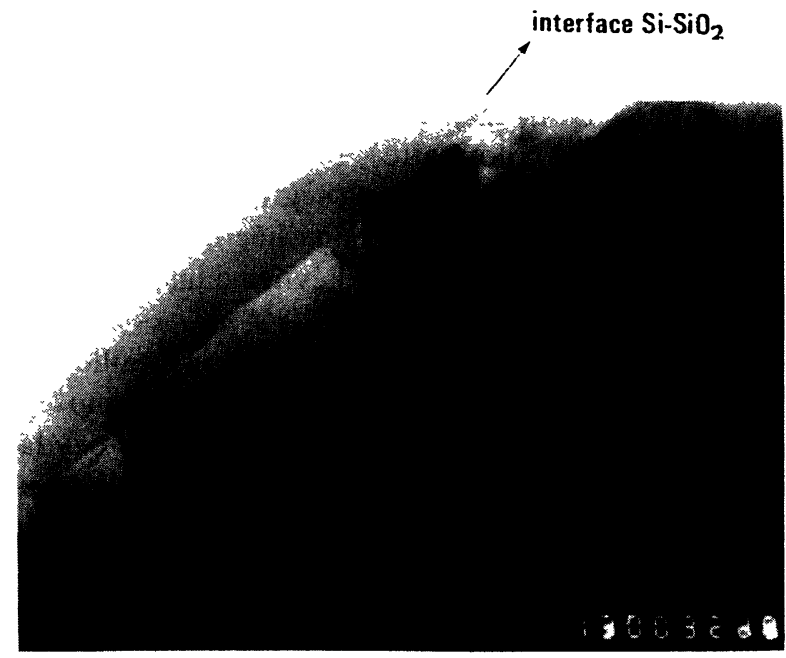

a)

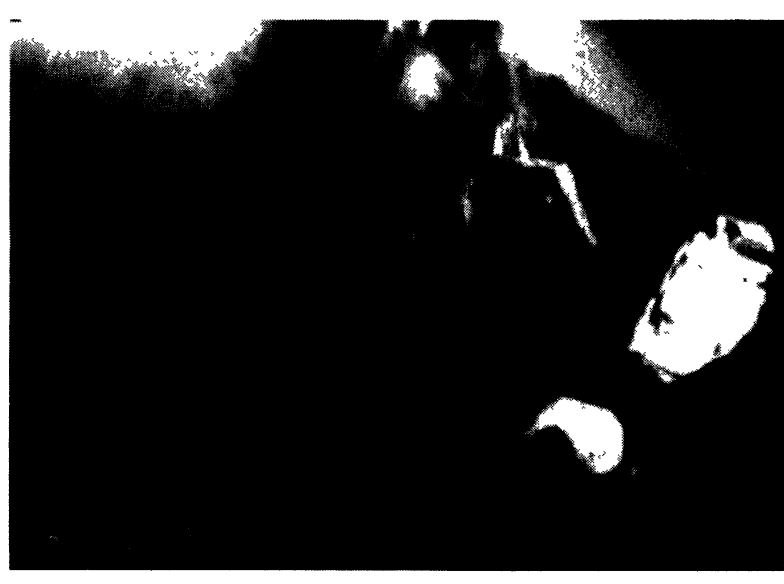

$14100 \mathrm{~nm} \sim 1$

b)

Fig. 3. - Observations en microscopie électronique par transmission: a) mise en évidence par M.E.T. de la rugosité de l'interface oxyde/silicium polycristallin après oxydation à $950^{\circ} \mathrm{C}$ pendant $1 \mathrm{~h}, \mathrm{~b}$ ) analyse microstructurale par M.E.T. du silicium polycristallin : mise en évidence de la grosseur des grains pour un échantillon oxydé à $1050^{\circ} \mathrm{C}$ pendant $30 \mathrm{~min}$.

[Transmission electron microscopy informations : a) T.E.M. results for oxide/polycrystalline silicon interface roughness after polysilicon oxidation at $950^{\circ} \mathrm{C}$ for $1 \mathrm{~h}$, b) T.E.M. microstructure analysis of polycrystalline silicon: we show the grain size of a sample oxidized at $1050^{\circ} \mathrm{C}$ for $\mathrm{min}$.]

lons non traités présentent des grains dont la taille est inférieure à $20 \mathrm{~nm}$ (de 18 à $19 \mathrm{~nm}$ ). Celle-ci évolue jusqu'à près de $70 \mathrm{~nm}$ après un traitement de recuit ou d'oxydation à haute température. Notons que cette taille initiale des grains est plus petite que celle d'échantillons de silicium polycristallin dopés par diffusion, à partir d'une source de $\mathrm{POCl}_{3}(50 \mathrm{~nm}$ environ $[13,18])$. Par ailleurs, la température de dopage par diffusion est nettement supérieure 
$\left(900{ }^{\circ} \mathrm{C}-1000^{\circ} \mathrm{C}\right)$ à la température du dopage in situ $\left(670^{\circ} \mathrm{C}\right)$, ce qui entraîne la présence de contraintes thermiques. De plus, dans les structures à gros grains (structure colonnaire), souvent la présence de canaux conducteurs favorise la ségrégation d'impuretés. La diminution de ces canaux liée conjointement à la taille des grains, permet d'éviter dans notre cas, ces inconvénients.

La figure 4 montre l'évolution de la taille des grains en fonction de la température et du temps de traitement, dans le cas d'un recuit et d'une oxydation. On note une différence sensible dans l'évolution de la taille des grains, suivant le type de traitement et particulièrement, pour des températures inférieures à $1000^{\circ} \mathrm{C}$. Pour ces températures $\left(<1000^{\circ} \mathrm{C}\right)$, le grossissement des grains est plus rapide lors d'une oxydation que lors d'un recuit.

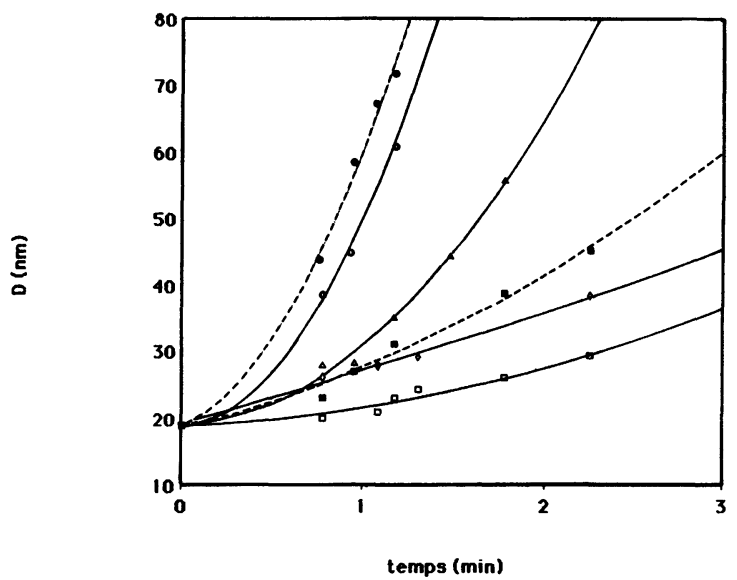

Fig. 4. - Influence de la température de recuit (-) (variant de $900^{\circ} \mathrm{C}(\square), 950^{\circ} \mathrm{C}(\diamond), 1000^{\circ} \mathrm{C}(\triangle)$ à $\left.1050^{\circ} \mathrm{C}(\mathrm{O})\right)$ et d'oxydation sous $\mathrm{O}_{2} \sec (---)\left(900^{\circ} \mathrm{C}(\square)\right.$ et $\left.1050^{\circ} \mathrm{C}(\bullet)\right)$ sur la taille moyenne des grains $D(\mathrm{en} \mathrm{nm})$ en fonction de la durée du traitement.

[Annealing temperature influence (一) (from $900{ }^{\circ} \mathrm{C}(\square)$, $950{ }^{\circ} \mathrm{C}(\diamond), 1000^{\circ} \mathrm{C}(\triangle)$ to $\left.1050^{\circ} \mathrm{C}(\mathrm{O})\right)$ and for dry $\mathrm{O}_{2}$ oxidation (---) $\left(900^{\circ} \mathrm{C}(\square)\right.$ and $\left.1050^{\circ} \mathrm{C}(\bullet)\right)$ on grain mean size ( $D$ in $\mathrm{nm})$ versus treatment duration.]

Ces observations nous permettent de proposer le mécanisme de croissance suivant :

1) le silicium polycristallin avant traitement de recuit ou d'oxydation, présente des zones amorphes et des petits grains (pic de diffraction large et de faible amplitude) ;

2) lorsque la température croît, quel que soit le type de traitement, ces zones amorphes cristallisent, ce qui conduit à l'amincissement des pics de diffraction. Cette cristallisation est favorisée, par ailleurs, par la présence de phosphore aux joints de grains. En effet, rappelons que le phosphore augmente la mobilité des atomes de silicium et favorise ainsi le réarrangement cristallin en gros grains ;
3) l'élévation de la température, dans tout mécanisme de diffusion, conduit à un grossissement des grains, et à une diminution des contraintes internes. Ces deux effets se traduisent respectivement, par l'affinement et le déplacement des raies de diffraction. Ces contraintes internes ont probablement pris naissance lors du dépôt CVD du silicium polycristallin, comme presque dans tous les dépôts CVD [26]. Elles sont remplacées, après un traitement thermique, par les contraintes liées au refroidissement. Celles-ci sont moindres, car le réarrangement des atomes à haute température, entraîne la disparition des zones amorphes responsables des contraintes mécaniques ;

4) le grossissement plus rapide des grains lors de l'oxydation peut être relié, comme dans le cas du silicium monocristallin $[19,27]$, à l'existence de contraintes interfaciales dues à l'oxyde. En effet, la différence entre les coefficients de dilatation thermique $\left(2,5 \times 10^{6}\right.$ pour $\mathrm{Si}$ et $0,5 \times 10^{-6}$ pour $\left.\mathrm{SiO}_{2}\right)$ entraîne, au niveau de l'interface $\mathrm{Si} / \mathrm{SiO}_{2}$, l'apparition de contraintes parallèles au plan interfacial, de compression dans l'oxyde, et de tension dans la couche de silicium polycristallin. Celles-ci peuvent, probablement comme dans le cas des métaux soumis à des contraintes, favoriser l'accroissement des grains du film polycristallin durant l'oxydation ;

5) l'écart, moindre à haute température, entre la taille des grains après un traitement sous Ar ou sous $\mathrm{O}_{2}$ (Fig. 4), peut s'expliquer par le fait que la masse volumique de $\mathrm{SiO}_{2}$, corps viscoélastique, dépend de $T$. Pour $T<960^{\circ} \mathrm{C}$, elle varie en fonction du temps jusqu'à une valeur limite. Si $T>960^{\circ} \mathrm{C}$, la masse volumique ne varie plus. Les contraintes mécaniques au niveau de l'interface sont alors plus faibles et leur influence devient négligeable. La cristallisation n'est plus favorisée que par les contraintes purement thermiques ;

6) lors d'un simple recuit une certaine quantité de phosphore est éliminée par exodiffusion dans la phase gazeuse, ce qui n'est pas le cas, en présence d'une couche d'oxyde. Cette plus forte concentration locale du dopant favorise alors, la croissance des grains et la vitesse d'oxydation.

\subsection{PROPRIÉTÉS ÉLECTRIQUES DES COUCHES. -}

Résistivité. - Les mesures de résistivité, obtenues à partir de la méthode des quatre pointes, sur des films de silicium polycristallin ayant subit des recuits sous Ar (Fig. 5), montrent que la résistivité des couches diminue en fonction de la durée du traitement thermique, conformément à l'hypothèse d'une migration des atomes de phosphore vers la surface du silicium polycristallin (exodiffusion en l'absence d'oxyde). Cette concentration du dopant à la surface est d'autant plus importante que la température du traitement augmente. On note, par exemple, une résistivité initiale de $9 \times 10^{-3} \Omega$.cm pour un échantil- 


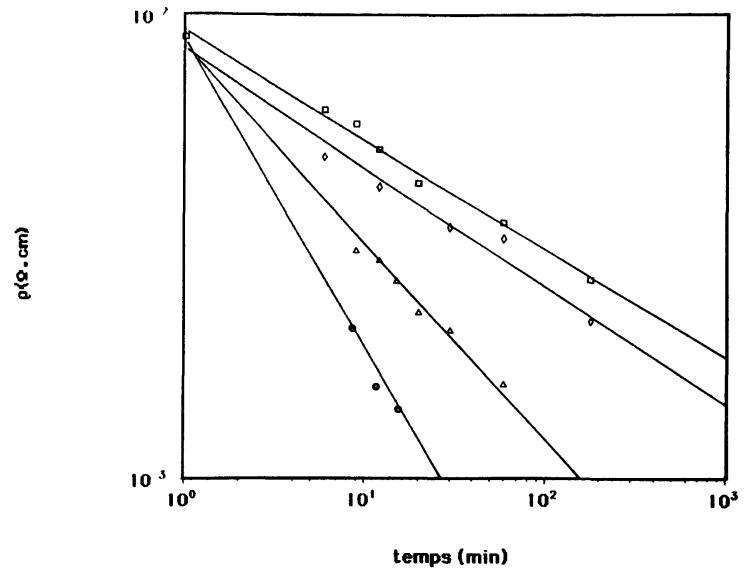

Fig. 5. - Variation de la résistivité de la couche de silicium polycristallin en fonction de la durée du traitement pour différentes températures de recuit $\left(p_{\mathrm{Si}}\right.$ vierge $=9 \times$ $\left.10^{-3} \Omega . \mathrm{cm}\right): \square=900^{\circ} \mathrm{C}, \diamond=950^{\circ} \mathrm{C}, \Delta=1000^{\circ} \mathrm{C}$, $\mathrm{O}=1050^{\circ} \mathrm{C}$.

[Polycrystalline silicon layer resistivity variation versus treatment duration for different annealing temperatures (virgin $p_{\mathrm{Si}}=9 \times 10^{-3} \Omega . \mathrm{cm}$ ) : $\square=900^{\circ} \mathrm{C}, \diamond=950^{\circ} \mathrm{C}$, $\square=1000^{\circ} \mathrm{C}, \mathrm{O}=1050{ }^{\circ} \mathrm{C}$.]

lon non traité. Celle-ci passe de $4,5 \times 10^{-3} \Omega . c m$ pour un échantillon recuit à $900^{\circ} \mathrm{C}$ pendant $30 \mathrm{~min}$, à près de $10^{-3} \Omega . \mathrm{cm}$ pour un échantillon traité durant le même temps à $1050^{\circ} \mathrm{C}$, soit une concentration en phosphore 2 fois, voire même 10 fois plus importante, par rapport à la concentration initiale. L'action du dopant (plus grande mobilité du silicium en présence de phosphore) a ainsi un effet catalytique, particulièrement à haute température $\left(>950^{\circ} \mathrm{C}\right)$, sur l'évolution de la taille des grains. Ces résultats sont en bon accord avec ceux trouvés par différents auteurs [22-24].

Rigidité diélectrique. - La caractérisation électrique des structures $\mathrm{M} / \mathrm{O} / \mathrm{Si}$-Polycristallin (équivalentes à des structures M.I.M.), montre que le principal mécanisme de conduction à travers l'oxyde, consiste en un phénomène de courant limité par charge d'espace [11]. La rigidité diélectrique (ou champ de claquage) est le rapport entre la tension provoquant la destruction définitive ou non du condensateur M.I.M. et l'épaisseur de diélectrique. Elle varie de $2 \mathrm{MV} / \mathrm{cm}$ à $5,4 \mathrm{MV} / \mathrm{cm}$ quand la température d'oxydation passe de $900^{\circ} \mathrm{C}$ à $1050^{\circ} \mathrm{C}$. Les structures qui présentent les meilleures propriétés isolantes sont celles oxydées à haute température (pour $\left.T=1050^{\circ} \mathrm{C}, E_{0}=5,4 \mathrm{MV} / \mathrm{cm}\right)$.

Les oxydes sur silicium polycristallin présentent des propriétés diélectriques moins bonnes que les oxydes sur silicium monocristallin, pour des conditions d'oxydation identiques. Cette différence est à relier à l'influence de divers facteurs sur la qualité «mécanique-électrique » de l'interface $\mathrm{SiO}_{2} /$ silicium polycristallin, produisant une fragilisation locale ou étendue de la couche d'oxyde, et se traduisant par l'existence de micro-claquages et un abaissement de la rigidité diélectrique.

L'oxydation intergranulaire est l'une des causes de cette «fragilisation électrique » de la couche. A basse température, lors de l'oxydation, la réaction de surface est le processus prédominant, l'espèce oxydante diffuse aux joints de grains et favorise l'oxydation intergranulaire. On observe, comme sur la photo 2 de la figure 3 , une couche d'oxyde présentant un aspect « ondulé » (réduction locale de l'épaisseur). L'existence de telles «micro-pointes» aux bords des grains, favorise l'accroissement local du champ électrique. Il en résulte le claquage primaire des structures, constaté pour un champ de l'ordre de $2,2 \mathrm{MV} / \mathrm{cm}$. A plus haute température, le phénomène de diffusion volumique prédomine (meilleure diffusivité d' $\mathrm{O}_{2}$ dans $\mathrm{SiO}_{2}$ ), ce qui minimise les effets d'oxydation intergranulaire et favorise une croissance plus homogène de l'oxyde et un interface silicium polycristallin $/ \mathrm{SiO}_{2}$ moins rugueux, avec des grains plus gros. Les micro-claquages sont dans ce cas, moins fréquents.

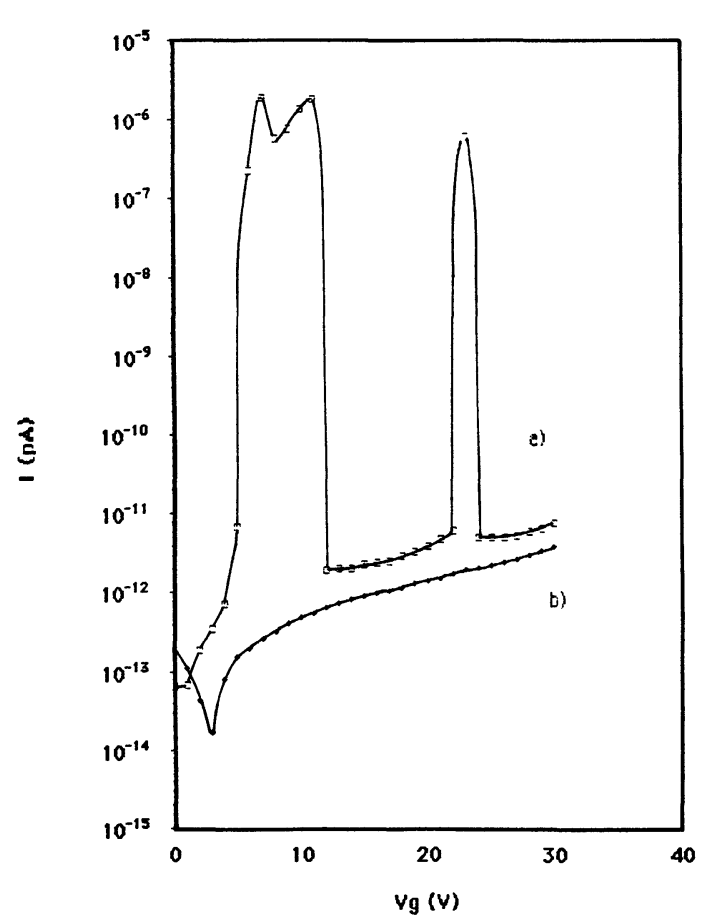

Fig. 6. - Variation du courant de conduction en fonction du champ électrique appliqué pour un échantillon oxydé à $900^{\circ} \mathrm{C}$ pendant $1 \mathrm{~h}$ : a) mise en évidence d'un microclaquage suivi d'un micro-recuit, lors d'un premier balayage en tension; b) mise en évidence de l'autoguérison après un deuxième passage.

[Current variation versus electric field for sample oxidized at $950^{\circ} \mathrm{C}$ during $1 \mathrm{~h} \mathrm{:} \mathrm{a)} \mathrm{pre-breakdown} \mathrm{obviousness} \mathrm{fol-}$ lowed by micro-annealing after a first applied voltage ; b) self-curring is observed after the second voltage sweeping.] 
La figure 6a donne un exemple d'évolution de la conductivité en fonction du champ électrique appliqué, pour un échantillon oxydé à $950^{\circ} \mathrm{C}$ pendant une heure. On note, une augmentation du courant de conduction dans le diélectrique jusqu'à $1 \mu \mathrm{A}$, puis une diminution brutale à quelques picoampères. Ceci traduit un micro-claquage, suivi d'un microrecuit. Le même phénomène se reproduit dans un autre domaine de tension. Lors d'un second «balayage » en tension (Fig. 6b), ce phénomène a totalement disparu. L'échantillon a subi un processus d'auto-guérison. La présence d'une protubérance au niveau des joints de certains grains, provoque lors de la mise sous tension, un effet de «pointe», se traduisant par l'apparition d'une région à faible résistance dans laquelle peut passer une forte intensité de courant. Le condensateur M.I.M. se décharge aussitôt à travers cette zone, ce qui provoque la «fusion locale» du diélectrique et de l'électrode supérieure. Le matériau diélectrique fondu, ainsi que la partie du dépôt de métal entourant le défaut sont rejetés sur la périphérie; il en résulte la "guérison" apparente de l'irrégularité de l'interface, qui se trouve ainsi isolée. Ce phénomène donne un «trou » observable par microscopie, de l'ordre de quelques dizaines de $\mu \mathrm{m}$ (Fig. 7), et entraîne une légère diminution de la surface de l'électrode. L'auto-guérison a lieu d'autant plus facilement que le métal s'évapore et s'oxyde avec

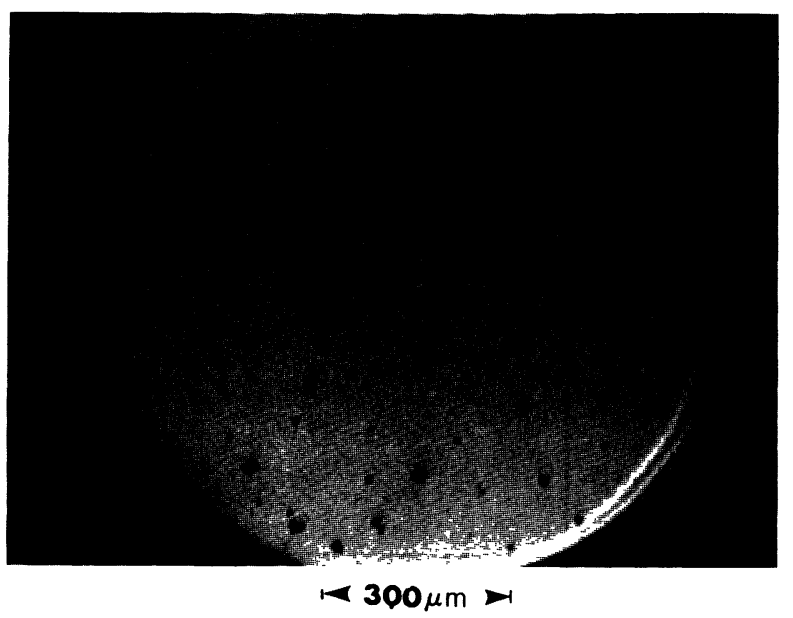

Fig. 7. - Observation par microscopie des trous dans l'électrode de grille de la structure M.I.M. après un microclaquage.

[Microscopic observation of holes in gate electrode device after a pre-breakdown.] une grande facilité à haute température, comme c'est le cas pour l'aluminium.

La meilleure tenue diélectrique est donc corrélée à un accroissement de la taille des grains, et une réduction de la « rugosité » interfaciale, c'est-à-dire du nombre des "pointes de champ », initiatrices du claquage diélectrique primaire, dégénérant en claquage destructif.

\section{Conclusion.}

La morphologie et la cristallinité des couches de silicium polycristallin sont très influencées par la nature des différents traitements thermiques qu'ils subissent. Nous avons comparé l'évolution des couches de silicium polycristallin soumises à des recuits sous argon ou à des oxydations sous $\mathrm{O}_{2}$ sec. Pour ce faire, nous avons utilisé une technique de diffraction des rayons $\mathrm{X}$. Celle-ci apparaît comme une très fine méthode d'analyse (bon accord avec les résultats obtenus par M.E.T.). Elle a permis d'étudier l'influence de la température d'oxydation sur la taille, et donc sur le nombre des grains. Conformément aux résultats obtenus par la diffraction des RX et par les mesures de résistivité, une plus forte concentration en phosphore dans le silicium polycristallin entraîne un accroissement de la taille des grains et produit une surface moins « rugueuse».

La cinétique d'oxydation du silicium polycristallin est principalement contrôlée par la présence des joints de grains et la ségrégation des dopants vers ces joints. A basse température, la vitesse de croissance de l'oxyde est contrôlée par une réaction de surface et dépend essentiellement de l'orientation aléatoire des grains. Il en résulte une « rugosité » de la surface de l'oxyde (fluctuations locales de l'épaisseur), accentuée par un plus grand nombre de grains. En dessous de $960^{\circ} \mathrm{C}$, les mécanismes associés à l'oxydation du silicium polycristallin sont :

(i) la diffusion intergranulaire de l'oxygène avec formation d'oxydes internes entre les grains ;

(ii) l'existence de contraintes mécaniques;

(iii) la non-uniformité de la couche d'oxyde (simple amincissement aux joints de grains, présence de protubérances) entraînant une «fragilité électrique ".

Par contre, à haute température (au-dessus de $960^{\circ} \mathrm{C}$ ), la diffusion volumique est le processus prédominant. En outre, il y a relaxation des contraintes mécaniques. Ces deux phénomènes favorisent une croissance plus homogène de l'oxyde et augmentent sa rigidité diélectrique. 


\section{Bibliographie}

[1] Nozawa, H., Matsunaga, J. and Hashimoto, K., 3th Symp. V.L.S.I. Sci. Technol. (Toronto) May 13-16 1985.

[2] IRENE, E. A., Thermal oxidation of silicon: New experimental results and models, Appl. Surf. Sci. 30 (1987) 1-16.

[3] Delarios, J. M., Helms, C. R., KaO, D. B. and DEAL, B. E., Effect of silicon surface cleaning procedures on oxidation kinetics and surface chemistry, Appl. Surf. Sci. 30 (1987) 17-24.

[4] WANG, Y.-Y. and ZHANG, A.-Z., The characteristics and morphology of oxidation of polycristalline silicon films in V.L.S.I., Proc. Adv. Semiconductor Technol. (1984) p. 139.

[5] Kamins, T. I. and McKenna, E. L., Met. Trans. 2 (1971) 2292.

[6] RIGo, S., Silica films on silicon, Instabilities in silicon devices, Silicon passivation and related instabilities, Eds G. Barbottin and A. Vapaille (Publ. by North-Holland, Amsterdam) 1 Chap. 1 (1986) 8107.

[7] Audisio, S., Lemiti, M., Bureau, J. C. and BalLAND, B., Elaboration à basse température de couches de $\mathrm{SiO}_{2}$ par dépôt chimique à partir d'une phase gazeuse, Bull. Soc. Fr. de Chimie 6 (1987) 125.

[8] IQbal, Z., Veprek, S., Webb, A. P. and CAPeZ ZUTO, P., Raman scattering from small particles size poly-Si, Solid State Commun. 37 (1981) 993.

[9] DupuY, M., Observations en microscopie électronique en transmission de coupes transversales minces, J. Micros. Spectros. Electron 9 (1984) 163

[10] Klug, H. P. and Alexander, L. E., X-Ray diffraction procedures (Publ. John Wiley, New York) 1954, chap. 9, p. 91.

[11] Huff, H. R., Halvorson, R. D., Chiu, T. L. and Guterman, D., Experimental Observations on Conduction through Polysilicon Oxide, J. Electrochem. Soc. 127 (1980) 2482-2488.

[12] Barnes, J. J., De Blasi, J. M. and Deal, B. E., Low Temperature Differential Oxidation for Double Polysilicon V.L.S.I. Devices, J. Electrochem. Soc. 126 (1979) 1779-1785.

[13] Shimada, K., Mori, S. and Mikata, Y., Reduction in Polysilicon Oxide Leakage Current by Annealing prior to Oxidation, J. Electrochem. Soc. 132 (1985) 2185

[14] HORIUCHI, M., Formation of thin oxides on poly-Si, J. Appl. Phys. 53 (1982) 155.
[15] Chen, D. N. and Chen, Y. C., A novel method for growing thin gate oxide, J. Electrochem. Soc. 132 (1985) 2510.

[16] Marcus, R. B. and Sheng, T. T., The oxydation of shaped silicon surface, J. Electrochem. Soc. 129 (1982) 1278

[17] Faraone, L., VibroneK, R. D. and McGinN, J. T., Characterization of Thermally oxidized $\mathrm{n}^{+}$polycristalline Silicon, IEEE Trans. Elec. Dev. vol. ED-32 (1985).

[18] Irene, E. A., Tierney, E. and Dong, D. W., Silicon oxydation studies : morphological aspect of the oxydation of polycristalline silicon, $J$. Electrochem. Soc. 127 (1980) 705-713.

[19] BALland, B., Defects in silica films. Their nature Their electrical properties, Instabilities in silicon devices, Silicon passivation and related instabilities, Eds G. Barbottin and A. Vapaille (Publ. North-Holland, Amsterdam) 1 chap. 2 (1986) 104-153.

[20] Camera Roda, G., SANTARelli, F. and SARti, G. C., A simplified viscoelastic model for the thermal growth of thin $\mathrm{SiO}_{2}$ films, J. Electrochem. Soc. 132 (1985) 1909.

[21] Eefnisse, E. P., Stress in thermal $\mathrm{SiO}_{2}$ during growth, Appl. Phys. Lett. 35 (1979) 245.

[22] SaRaswat, K. C. and Singh, H., Thermal Oxidation of Heavily Phosphorus-Doped Thin Films of Polycristalline Silicon, J. Electrochem. Soc. 129 (1982) 2321

[23] Kamins, T. I., Structure and Properties of LPCVD Silicon Films, J. Electrochem. Soc. 127 (1980) 686.

[24] Mandurah, M. M., Sarawsat, K. C. and Kamins, T. I., Phosphorus Doping of Low Pressure Chemically Vapor-Deposited Silicon Films, J. Electrochem. Soc. 126 (1979) 1079.

[25] Kern, W. and Rosler, R. S., Advances in deposition processes for passivation films, J. Vac. Sci. Technol. $24 \mathrm{n}^{\circ} 5$ (1977).

[26] Audisio, S., Mai, C., Monnier, G., Schaeffer, G. and R. RIVIÈRE, Sur l'obtention de couches minces de siliciures de fer par CVD et les études des contraintes, de frottement sec et de l'usure des échantillons siliciés, Bull. Soc. Chim. France 3 (1971) 742-747.

[27] LEROY, B., Stresses and silicon interstitiels during the oxydation of a silicon substrate, Phil. Mag. $B$ 55, 2 (1987) 159-199. 